\title{
KNOWLEDGE REPRESENTATION APPROACH TO CLOSED LOOP CONTROL SYSTEM - A TANK SYSTEM CASE-STUDY
}

\author{
Luís Rato, Irene Pimenta Rodrigues, Rui Gomes \\ Universidade de Évora \\ R. Romão Ramalho, 59, 7000-671 Évora \\ Email: $\{$ lmr,ipr\}@di.uevora.pt
}

Keywords: Control, declarative programming, knowledge representation, constraints.

\begin{abstract}
Control engineering problems are dealt within a plethora of methods and approaches depending on the a priori knowledge, the description of the process to control, and the main control goal. Classical control theory is mainly based on properties of numerical models. This paper presents an approach that applies to a class of processes described by numerical and logical relations using inference and a knowledge base system. To attain this goal an ontology for control systems is constructed. The work presented in this paper is based in a three tank system benchmark.
\end{abstract}

\section{INTRODUCTION}

The knowledge representation as a major impact on the performance of intelligent systems (Messina et al., 2001) and the relevance of this fact increases with the complexity of systems we are dealing with. Thus, considering the diversity and the potential complexity of control engineering problems one must consider that control systems are a natural candidate to apply knowledge bases and inference systems.

Most applications of artificial intelligence algorithms to control problems amount to softcomputing techniques and supervision tasks (Shadbolt et al., 1989). Nevertheless, the concern on modelization from the control engineering community has produced some work on component oriented model language as in the Modelica language (Elmqvist et al., 2001) or CSML (Zagar et al., 2001). In this work we aim to describe, infer the behaviour, and infer control actions in a integrated way through a Prolog implementation using finite domain constraints (Diaz and Codognet, 2000; Sterling and Shapiro, 1997).

Although the importance of knowledge representation is recognised and Prolog is used to express declarative rules about controller properties in (Shadbolt et al., 1989), the inference system remains limited to supervision tasks. This work presents a more integrated approach from process description to control tasks and aims to be as general as possible.

The scope broadening of such approach will be at- tained with a penalty in efficiency when compared to the very powerfull classical linear system tools. Thus, in order to illustrate the potential of such approach a non linear, multi-input multi-output (MIMO) system is chosen as a test-case. For such systems the powerfull mathematical tools are restricted to very particular cases. Another possible approach is to combine integer programming with real constraint optimisation (Bemporad and Morari, 1999). Nevertheless, such tools do not have a programming framework to support them as powerful as logic programming has. The test-case used in this work, a three-tank system, has been used as a very flexible benchmark system for reconfigurable control techniques (Lunze et al., 2001).

This paper is organised as follows. An introduction is made in section 1 . Section 2 presents the knowledge based control system build around the three tank system test-case. The model is validated by simulation in Section 3. Section 4 presents the queries that solve the proposed control problems. Some conclusion are addressed in Section 5.

\section{Knowledge based control system}

In this section a knowledge based control system is build around the three tank system test-case. Though the knowledge based system presented here does not aim to a general purpose solution to process modelling and control it presents solutions that can be eas- 
ily extended to other processes and control problems.

Some of the control problems that we aim to address are: estimation, fault detection and isolation, control actuation, and controller optimisation. These control problems can be define in a declarative way according to:

- Estimation - given a series of observations of the process which are the possible internal states at instant $t$.

- Fault detection and diagnosis - given a series of observations of the process which are the admissible models or model variations.

- Control actuation - given a series of observations and a control goal (control reference) which should be the actuation on the process such that the goal is attained at an instant $t$.

- Controller optimisation - given process and controller models which are the controller parameterers such that the closed loop system is optimal according to a criteria (penalty function).

These set of classical control problems may be defined with constraints such as internal state variables, solution space, and time constraints. This fact suggests the use of a constraint oriented programing tool.

\subsection{Linear dynamical system}

In order to illustrate the potential of the proposed approach consider a discrete-time linear dynamical system to be represented in logic programming with constraints. Discrete-time is considered since the GnuProlog constraint system applies only to integer domain.

$$
\begin{gathered}
x(t+1)=A x(t)+B u(t) \\
y(t)=C x(t)+D u(t)
\end{gathered}
$$

where $x(t)$ is the state; $u(t)$ is the input; $y(t)$ is the output and $A, B, C, D$ are matrices which parameterise the linear system. The following problems will be considered:

- Simulation - given parameters A,B,C,D, the input $u(t)$ and initial conditions $x(0)$ what is the state $x$ or output $y$ at a certain instant $t$

- Input definition - given parameters A,B,C,D, the desired output $y$ at time $t$ (with an error margin $\delta$ ), initial conditions $x(0)$ what are the possible inputs $u(t)$ (with some constaints, e.g. constant input).

- Parameterisation - given parameters A,B,C,D, partially defined, the input $u(t)$ and initial conditions $x(0)$ what should be the parameters such that the output $y$ is the desired one at time $t$ (with an error $\operatorname{margin} \delta$ )

\section{Prolog Implementation}

Two predicates are defined to implement the state and output equations. The predicate "state" defines the state $x(t+1)$ recursively

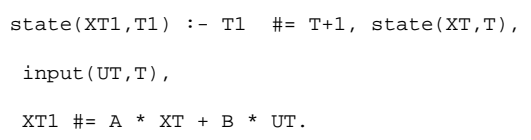

and the predicate "output" defines the output $y(t)$

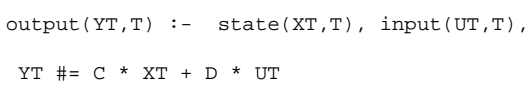

where $A, B, C, D$ stands for the linear system parameters; $X T$ is the state $x(t), X T$ is the input $u(t)$ and $Y T$ is output $Y T$. It should be noted that, in general, the operators "*" and "+" represent the matrix product and sum, and the variables $X T, Y T$ and $U T$ are vectors with compatible dimensions. Furthermore, since GNU-Prolog constraint solver operates only on integer positive values, operators "*" and "+" and number representation must be such that positive and negative limited precision real values are represented through integer positive values.

To attain this goal negative integer are represented using tens complements. This is a particular case of a modular arithmetic (modulo a ten's power) (Knuth, 1997), just as two's complements in computer arithmetic. In order to represent decimal part, variables are scaled by a constant $10^{n}$.

Initial contitions may be introduced through a fact such as

state $(0,0)$.

which stands for $x(0)=0$ in a first order system.

Consider the simulation problem. Given zero initial conditions, and constant input $u(t)=1$, the value $X T$ unifies to 1 for any time $T$.

input $(1,-)$.

the following query defines the output at instant 100 .

output (YT, 100).

The second proposed problem is input definition. Given a desired output $y=$ yout with a certain tolerance $t o l$, at time $t=100$ what should be the input, if it is constant.

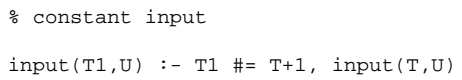

the query could be defined as

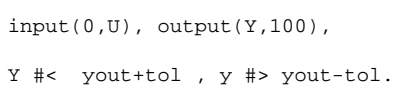

where yout and tol are the desired output value and tolerance; and $U$ is the variable that unifies with a solution. 


\subsection{Tank System case-study}

The three tank system, in figure 1, is a rich benchmark system due to its nonlinear behaviour, actuators and sensors which can be continuous as well as on-off, and redundancy which allows to test reconfigurable control strategies(Rato and Lemos, 1999). Control reconfiguration is a control problem usually tackled by non classical control tools, thus a good candidate to illustrate the potential of a knowledge based approach.

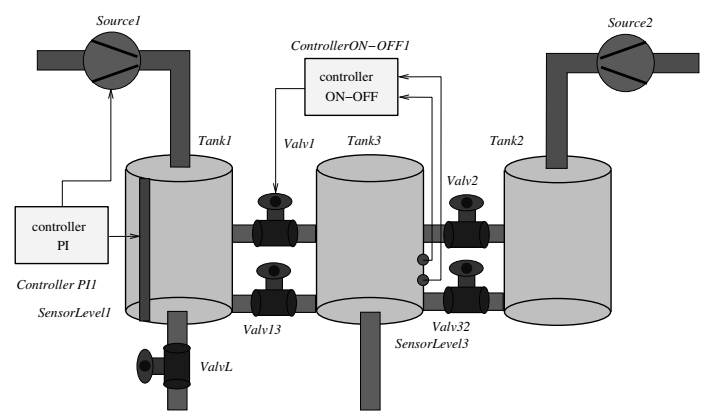

Figure 1: Tree tank system

The mathematical model of tank systems are represented by the following differential equation for each tank,

$$
\dot{h}(t)=\sum_{i=1}^{N} \frac{Q_{i}(t)}{A_{T}}
$$

where $A_{T}$ is the area of the tank base's, $N$ is the number of inputs/outputs, and $Q_{i}$ is the inflow in each tank's input/output. External sources are manipulated variables.

$$
Q_{i}(t)=u(t)
$$

and whenever there is a pipe at height $h_{p}$ (only constant section horizontal pipes are considered in this work) between two tanks the inflow is defined by: if $h>h_{p}$ and $h^{\prime}>h_{p}$

$$
Q_{i}(t)=a_{Z} \operatorname{Sggn}\left(h^{\prime}-h\right) \sqrt{2 g\left|h^{\prime}-h\right|}
$$

if $h>h_{p}$ and $h^{\prime}<h_{p}$

$$
Q_{i}(t)=a_{Z} \operatorname{Ssgn}\left(h^{\prime}-h_{p}\right) \sqrt{2 g\left|h^{\prime}-h_{p}\right|}
$$

if $h<h_{p}$ and $h^{\prime}>h_{p}$

$$
Q_{i}(t)=a_{Z} \operatorname{Ssgn}\left(h-h_{p}\right) \sqrt{2 g\left|h-h_{p}\right|}
$$

if $h<h_{p}$ and $h^{\prime}<h_{p}$

$$
Q_{i}(t)=0
$$

where $h$ is the level of the tank being modelled; $h^{\prime}$ is the level of another tank connected at height $h_{p}$.

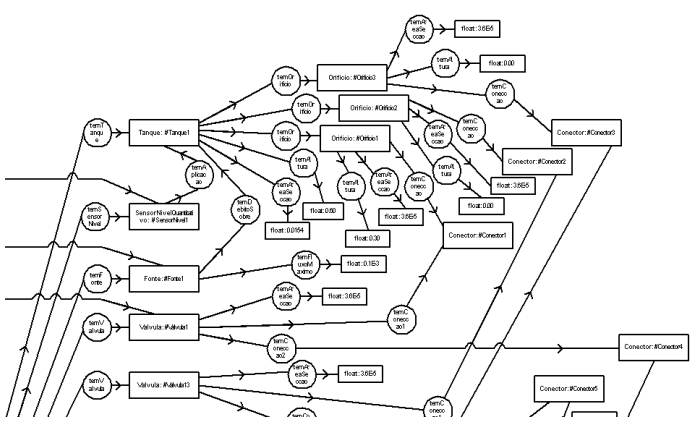

Figure 2: Catalog of individuals

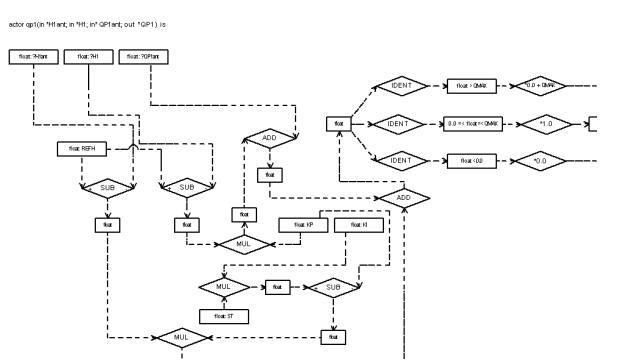

Figure 3: Model representation

The three tank system is composed by three tanks $6 m$ high, with a section surface $S=3.610^{-5} \mathrm{~m}^{2}$, with 2 pipes connecting the central tank to each one of the other two tanks - one at $h=0$ and another at $h=0.3 m$ - each pipe with section surface $A=0.0154 \mathrm{~m}^{2}$ has a valve and there are two pumps that put water into tanks 1 and 2. Maximum flow for each pump is $Q_{\max }=0.110^{-3} \mathrm{~ms}^{-1}$ Constant $a_{z}$ is assumed unitary. The level of the tanks can be controlled using the flow from the pumps as well as through the valves.

A complete model description may be found in (Lunze et al., 2001). The continuous time model is obtained discretizing the continuous model using Euler's method (Åström and Wittenmark, 1997).

\subsection{Three tanks representation as a Finite Domain Constraint Satisfaction Problem}

To represent the three tanks systems as a constraint satisfaction problem in Prolog we had to build an ontology in order to represent the system entities and its proprieties.

In figure 2 we present a excerpt of the defined conceptual graph for representing our domain ontology (three tanks problem). In figure 4 we present the description of an example according to the ontology defined in figure 2. 


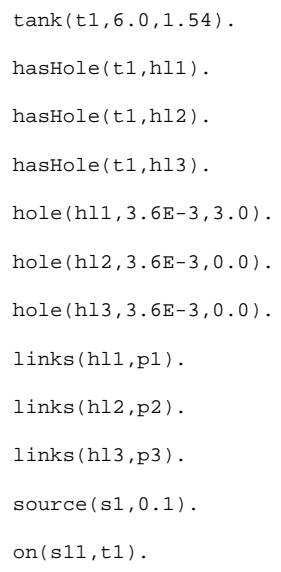

Figure 4: Representation of a three thanks configuration

In Prolog with finite domain constraints our entities may be represented by: names, constants values, variables or constraint variables. Proprieties are represented by predicates that relate one or more entities.

In this paper we will detail the representation of the proprieties:

- Tank level height

In our ontology tanks have the propriety of having a level hight at a time instant, this is represented by the 3 arguments predicate $h$ where the first argument represents the tank name, the second one is a constraint variable that represents the tank hight and the third one is a constraint variable that represents a time instant

Example: h(t1,HT,T), represents that tank $\mathrm{t} 1$ has height HT at the time instant T.

The height value, as we can see from equation ??, at a time instant depends on the flow of the pipes or sources connected to the tank.

- Pipes and sources flow.

Pipes are connected to tanks and have the propertie of having a flow at a time instant. The predicate to represent the flow of a pipe or a source is a 3 arguments predicate $q$ where the first argument represents the pipe or source name, the second one is a constraint variable that represents the pipe flow and the third one is a constraint variable that represents a time instant.

\section{Example:}

$\mathrm{q}(\mathrm{p} 1, \mathrm{QT}, \mathrm{T})$, represents that pipe $\mathrm{p} 1$ has flow QT at the time instant $\mathrm{T}$.

$\mathrm{q}(\mathrm{s} 1, \mathrm{QT}, \mathrm{T})$, represents that source s1 has flow QT at the time instant $\mathrm{T}$.

In order to model the behaviour of three tanks represented in figure 1 we had to define a set of rules such has the following ones:
- rule to model Tank t1 level height

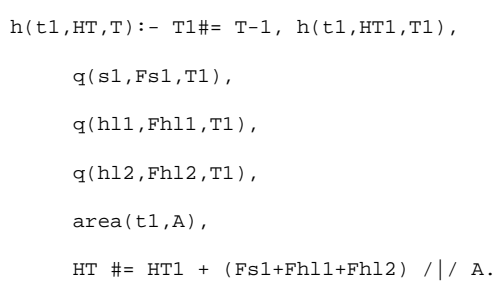

The operator // - represents the integer division between integer represented in ten-complement.

Flow and height values are represented in tencomplement since flows in pipes may be positive or negative.

- rule to model Tank1 t1 initial conditions

Supposing that the initial conditions where that thank 1 has a level less then 10until 5 seconds. The following rule represents this initial conditions.

$h(t 1, H, T):-H \#<10, T \#<5$.

- rule to model flow in source $\mathrm{s} 1$

The following rule states that source $\mathrm{s} 1$ has a flow of 20 from $t=0$ until $t=10$.

$\mathrm{q}(\mathrm{S} 1, \mathrm{FT}, \mathrm{T}):-\mathrm{FT} \#=20, \mathrm{~T} \#>=0, \mathrm{TH}=10$.

- rule to model flow in pipe $\mathrm{p} 1$.

This rule will be obtain from the general equations presented in the previous subsection taking into account that pipe $\mathrm{p} 1$ connects tank $\mathrm{t} 1$ and tank $\mathrm{t} 2$.

For instance the translation of the rule:

$$
\begin{aligned}
& \text { if } h>h_{p} \text { and } h^{\prime}>h_{p} \\
& \quad Q_{i}(t)=a_{Z} S \operatorname{sgn}\left(h^{\prime}-h\right) \sqrt{2 g\left|h^{\prime}-h\right|} \\
& \mathrm{q}(\mathrm{P} 1, \mathrm{FT}, \mathrm{T}):-\mathrm{T} 1 \text { \#= T-1, } \\
& \text { h(t1, HT1, T1), } \\
& \text { h(t2, HT2, T1), } \\
& \text { height (P1, H), } \\
& \text { HT1\#>H, HT2\#>H, } \\
& \text { HD\#= HT1-HT, } \\
& \text { module (HD, HDM), } \\
& \text { FT1*FT1 \#=2* 10*HDM, } \\
& \text { signal (HD, FT1, FT). }
\end{aligned}
$$

\section{Control and diagnosis queries}

In this section we present some examples in order to illustrate the results of our modulation of the three tanks using logic programing with finite domain constraints. 


\subsection{Model validation}

The generated declarative model of the tank system is validated comparing simulation results in figure 5 with results from a MATLAB implemented model according to (Lunze et al., 2001), in figure 6

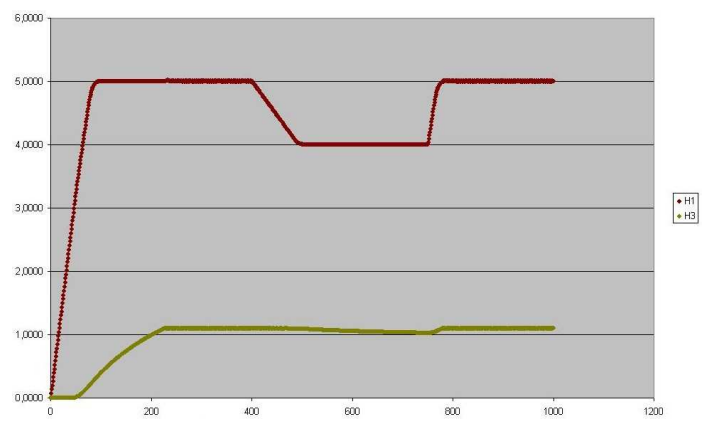

Figure 5: Three tank system simulation - GNUProlog

The results of the generated declarative model implemented in PROLOG with finite domain constraints are according to those from the MATLAB simulation.

\subsection{Example 1 - A diagnosis problem}

Consider the tank system and admit that a leakage may occur. This fault is simulated by opening of valve $1 L$ which is in the bottom of tank 1 , at instant $t=160$. One may wish to find in which instants such a fault may occur considering the observations. This amounts to a diagnosis problem.

The following constraints were considered: the fault occurs in the interval $150 s<t<175 \mathrm{~s}$; the model is admissible if the simulated tank level $h_{s}$ imul and the observations are such that $\mid h_{\text {simul }}-$ $h_{o b s} \mid<10 \mathrm{~mm}$. The query is,

| ?- findall (T, (T\#<300,

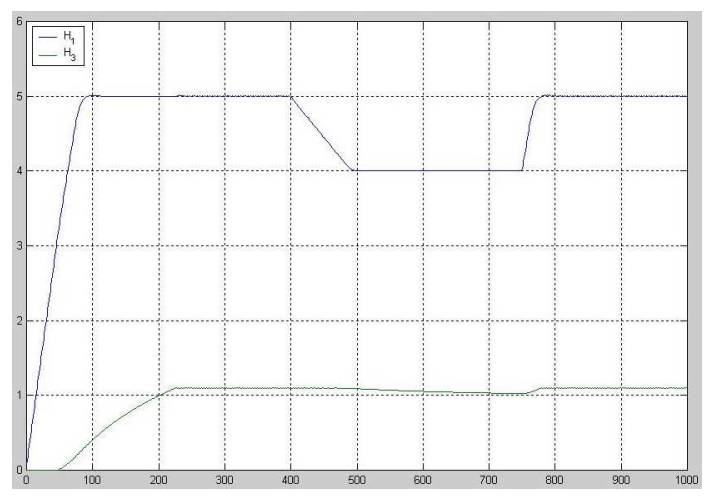

Figure 6: Three tank system simulation - MATLAB

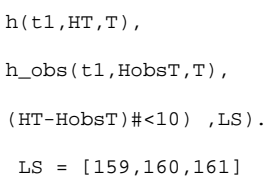

Where the predicate $h_{-}$obs is in the database defined for all relevant time instants, 0 to 300 . then,

If the solutions are ordered by decreasing error

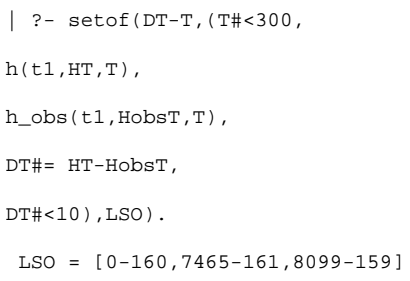

the instant $t=160$ is the most likely to be the correct answer. It should be noticed that in this simulations there is not addition of noise on sensor signals so, exact answers are found.

\subsection{Example 2 - control problem with constraints}

Consider the goal is to have the tank level at $205 \mathrm{~mm}$ at instant $t=60 \mathrm{~s}$. We should define the input flow $Q$ (from a pump) considering this flow to be constant in the interval $0 s<t<60 s$ with the constraint $75000 \mathrm{~mm} / \mathrm{s}<Q<80000 \mathrm{~mm} / \mathrm{s}$.

This constraints could be stated by the following rules:

$\mathrm{q}(\mathrm{S} 1, \mathrm{FT}, \mathrm{T}):-\mathrm{FT}<<80000, \mathrm{FT} \#>75000, \mathrm{TH}<60, \mathrm{TH}>60$.

$h(t 1,205, T):-$ T\#=60, ! .

This amounts to a control problem with constraints, whose solution is obtained by the query,

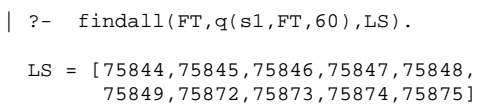

where the predicate $h(t 1, H T, T), T \#=60$ calculates the tank level at $t=60 \mathrm{~s}$. This results depends on the initial conditions of the tank system. Thus, one of those solutions can be applied in a received strategy. Which means that after the solution is obtained and applied, new observations are made and a new solution is calculated.

\subsection{Example 3 - calculating optimal gains}

Consider the tank water level in closed-loop controlled by a fixed structure controller such as a PI controller. We want to determine the controller parameters under the following constraints: the controller gains (proportional gain $k_{p}$ and integral gain $k_{i}$ ) are in the intervals $1<k_{i}<10$ and $10<k_{p}<100$; the 
tracking error (the difference between the goal and the actual level) is bounded to $5 \mathrm{~mm}$ ref $-h \mid=<5 \mathrm{~mm}$; and there is no overflow in the tank $h<600 \mathrm{~mm}$ within the following time interval $25 s<t<100 s$. which is solved by the following query, whose set of solutions is over 200.

| ?- findall((KP,KI), sim(KP,KI,_), LS).

$\mathrm{LS}=[(25,2),(26,2),(27,2),(30,3), \ldots]$

where $\operatorname{sim}\left(K P, K I, E R R_{-} T O T A L\right)$ is a predicate that calculates the tracking error of the closed loop system with gains $K_{i}$ and $K_{p}$.

An optimal parameterisation is obtained with a increasing error ordered list

| ?- setof (ET- (KP, KI), sim (KP, KI, ET), LSO).

LSO $=[3330-(75,10), 3337-(74,10), \ldots]$

resulting in the optimal gains $K_{i}=10$ and $K_{p}=75$.

\section{Conclusions and Future Work}

A declarative approach to control problems that applies to a class of processes described by numerical and logical relations using inference and a knowledge base system as been presented. Logic programming with constraints has been applied. The followed approach makes possible not only to represent the control system in declarative way but also to query the knowledge base system using the same declarative language. The complete system is described using the same description language no matter we are dealing with logical or numerical variables. A further step in the research will be to explore constraint logic programming using other solvers that cope with real variables (positive or negative) possibly using mixed integer quadratic optimisation and compare the precision gains versus the computational complexity increase. Another problem that should be carefully analysed, in the presence of quantisation, is the propagation of quantisation error across the inference process.

\section{REFERENCES}

Åström, K. and Wittenmark, B. (1997). Computer Controlled Systems. Prentice-Hall.

Bemporad, A. and Morari, M. (1999). Control of systems integrating logic, dynamics, and constraints. Automatica.

Diaz, D. and Codognet, P. (2000). The gnu prolog system and its implementation. In ACM Symposium on Applied Computing-SAC, Villa Olmo, Como, Italy. ACM.

Elmqvist, H., Mattson, S., Otter, M., and Astrm, K. (2001). Modeling complex systems. In Astrm, K., Albertos, P., Isidori, A., Schaufelberger, W., and Sanz, R., editors, Control of Complex Systems, chapter 2. Springer.
Knuth, D. (1997). The Art of Computer Programming Seminumerical Algorithms. Addison-Wesley.

Lunze, J., Askari-Marnani, J., Cela, A., Rato, L., Lemos, J., and Staroswiecki, M. (2001). Three-tank control reconfiguration. In Astrm, K., Albertos, P., Isidori, A., Schaufelberger, W., and Sanz, R., editors, Control of Complex Systems, chapter 2. Springer.

Messina, E. R., Evans, J. M., and Albus, J. S. (2001). Evaluating knowledge and representation for intelligent control. In 2001 PerMIS Workshop, Mexico.

Rato, L. and Lemos, J. M. (1999). Multimodel based fault tolerant control of the 3-tank system. In Proceedings of European Control Conference ECC99, Karlsruhe, Germany.

Shadbolt, N. R., Robinson, B. D., and Stobart, R. K. (1989). knowledge represebtation for closed loop control. In SIGART: ACM Special interest group on AI. ACM Press.

Sterling, L. and Shapiro, E. (1997). The Art of Prolog. MIT Press.

Zagar, K., Plesko, M., G.Tkacik, and Vodovnnik, A. (2001). The control system modelling language. In ICALEPCS-2002, San Jose CA. 